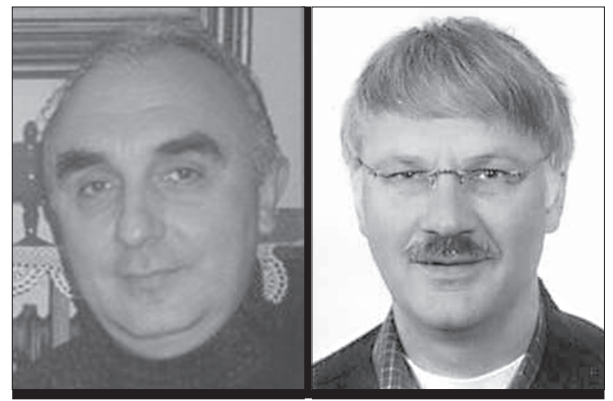

George Pavlou

\section{Network and Service Management}

his is the third issue of the series on Network and Service Management that is published twice a year. The series provides articles on the latest developments in this wellestablished discipline, highlighting recent research achievements and providing insight into both theoretical and practical issues related to the evolution of the discipline from different perspectives. The series provides a forum for the publication of both academic and industrial research, addressing the state of the art, theory, and practice in network and service management.

In the last editorial we mentioned that the recently established IEEE Electronic Transactions on Network and Service Management (eTNSM) had passed the first major IEEE approval, in which the proposed title, scope, and sponsorship were approved. More recently, the next and final key milestone was passed, which means that IEEE eTNSM will become a full-fledged IEEE transactions/journal from January 2007; see the editorial in the latest issue by the Editor in Chief Prof. Raouf Boutaba: http://www.comsoc.org/livepubs/etn/public/ 2006/apr/editor.html. The journal currently publishes two issues per year, and five issues have been published so far. These are freely available at present at http://www.comsoc.org/ eTNSM.

Another important development for the community has been the start in the beginning of 2006 of the European EMANICS project on Management of the Internet and Complex Services. This is an EU Network of Excellence that brings together 13 research institutions active in the area of network and service management. It encompasses work areas dealing with integration (long-term vision, virtual laboratory, and testbeds), dissemination (a new European conference, electronic dissemination, training and technology transfer, open source initiatives), and joint research activities (scalable, economic, and autonomic management). For more information, visit the project site: http://www.emanics.org/. You will also find there a newsletter with community news, events, and developments that is published three times a year and to which you may subscribe if you want to receive it in "push" mode.

Finally, it is worth noting that a key annual event in this area, the 2nd International Week on the Management of Networks and Services (ManWeek 2006), is to be held October 23-27, 2006 in Dublin, Ireland. This includes the well-established events MMNS, DSOM, and IPOM, and also the workshops MASE and AGNM; http://www.manweek2006.org/. Also this year there was for the first time an Integrated Network Management (INM) workshop attached to ACM SIGCOMM that took place on September 11, 2006 in Pisa Italy.
We again experienced overwhelming interest in the third issue, receiving 17 submissions. All these submissions were subject to three independent reviews, and we finally selected four articles, resulting in an acceptance rate of $23.5 \%$. Reviews for the first article in which the first Series Editor, Prof. Pavlou, is a co-author were independently handled by the second series editor, Dr. Pras. We intend to maintain our rigorous review process in future issues, thus maintaining the high quality of the published articles.

The first article, "A Methodological Approach toward the Refinement Problem in Policy-Based Management Systems" by Rubio-Loyola, Serrat, Charalambides, Flegkas, and Pavlou, provides first an introduction to policy refinement and the importance of establishing policy hierarchies, and then presents a relevant methodology that is validated through a holistic scenario applied to the quality of service management domain.

The second article, "Decentralized Service Level Monitoring Using Network Threshold Crossing Alerts" by Wuhib, Stadler, and Clemm, presents a network monitoring architecture and protocol in which information is aggregated across managed devices, providing the capability to implement in real-time network-wide threshold crossing alerts. Applicability examples are also presented.

The third article, "Managing Fast Aggregation Networks for Fast Moving Users" by Van Quickenborne, De Greve, De Turck, and Demeester, argues that a hierarchical wired Ethernet aggregation network together with Ethernet-based wireless access networks is a suitable approach for providing broadband access to fast moving users (e.g., in trains).

Finally, the fourth article, "Managing Agility through Service Orientation in an Open Telecommunication Value Chain" by Bastiaansen and Hermans, describes a strategy for managing commercial and operational agility in an open telecommunication value chain, focusing on openness and reusability for providing such agility in deregulated and competitive telecommunication environments.

We hope that readers of this issue find again the articles informative, and we will endeavor to continue with similar issues in the future. We would finally like to thank all the authors who submitted articles to this series, and the reviewers for their valuable feedback and comments on the articles.

\section{BIOGRAPHIES}

GeORGe PAVlou (G.Pavlou@surrey.ac.uk) is a professor of communication and information systems at the Center for Communication Systems Research, Department of Electrical Engineering, University of Surrey, United Kingdom, where he leads the activities of the Networks Research Group. He received a 
Diploma in electrical and mechanical engineering from the National Technical University of Athens, Greece, and M.Sc. and Ph.D. degrees in computer science from University College London, United Kingdom. His research interests focus on network management, networking and service engineering, including policy-based management, programmable networks, traffic engineering, multimedia service control, and object-oriented communications middleware. He has been instrumental in a number of European and U.K. research projects, and has contributed to standardization activities in ISO, ITU-T, and IETF. He was technical program co-chair of the Seventh IFIP/IEEE Integrated Management Symposium (IM 2001).

AIKo PRAs (pras@cs.utwente.nl) is an associate professor in the Departments of Electrical Engineering and Computer Science at the University of Twente, the Netherlands and a member of the Design and Analysis of Communication Systems Group. He received a Ph.D. degree from the same university for his thesis titled Network Management Architectures His research interests include network management technologies, Web services, network measurements, and accounting. He has participated in many European and Dutch research projects, such as SURFnet6 RoN, M2C, WASP, and Internet NG. He currently is Research Leader in the European Network of Excellence on Management of the Internet and Complex Services (EMANICS). He has also contributed to research and standardization activities as a member of the Internet Research Task Force (IRTF) Network Management Research Group (NMRG). He was technical program co-chair of the Ninth IFIP/IEEE Integrated Management Symposium (IM 2005).

\section{IEEE Communications MAgaZine \\ CALL FOR PAPERS \\ Wireless Broadband Access - WiMax AND Beyond}

Recent developments on wireless communication technology have resulted in tremendous innovations to make wireless access networks able to replace the wired access networks with much more bandwidth. The IEEE $802.16 \mathrm{~d}$ standard, which has been ratified in June 2004, has specified all the techniques needed at the physical layer, and the services that should be provided at the medium access control (MAC) layer in the WiMax systems. As a wireless access network, WiMax has shown great potential to provide broadband transmission services to the residential houses. The services include normal Internet access, data transmission, as well as voice and video transmission due to its broad bandwidth. With the support of mesh networking, WiMax systems can be easily configured as a wireless metropolitan area networks (WMAN). The IEEE 802.16e, which was officially approved in December 2005, has further enhanced the ability of WMANs with mobility support. The amended standard specifies the mobile WMANs for combined fixed and mobile broad bandwidth access supporting subscriber stations moving at vehicular speeds operating in licensed bands below $6 \mathrm{GHz}$. The IEEE 802.16e standard provides service providers the ability to offer a wide range of new and revolutionary high-speed, mobile wireless applications and services that will greatly improve people's way of life. It can be expected that with the mobile WMANs, much more flexibility and applications will be provided in our modern life to achieve the goal of accessing the global information at any place and at any time by any mobile device in the future. The significance of the WMAN development is obvious and huge.

The aim of this Feature Topic is to feature the recent advances in theory, application and implementation of WiMax networks including mobile WMANs. Articles as both research papers and tutorials from both academia and industry are solicited. Topics of interests include, but are not limited to:

- Enhancing techniques for the physical layer of WiMax systems

- QoS service enhancements for the medium access control layer

- Topology and architecture of WiMax mesh networks

- Medium access control and routing issues in WiMax mesh networks

- Introduction of mobile WMANs

- Security architectures and protocols for WiMax mesh networks and mobile WMANs

- Novel application and deployment scenarios

- Standardization activities

- Practical issues including performance measurements, deployment, field trials on scalability in dense areas, interference, coverage and mobility

- Comparative studies with other competing solutions such as IEEE 802.20 standard

- WiMax system design issues

Articles should be tutorial in nature and written in a style comprehensible to readers outside the specialty of the article. Mathematical equations should be minimal. Articles should not exceed 4500 words. Figures and tables should be limited to a combined total of six. Complete guidelines for prospective authors can be found at:http://www.comsoc.org/pubs/commag/sub_guidelines.html. Please submit PDF (preferred) or MSWORD formatted papers by the submission deadline to Manuscript Central (http://commagieee.manuscriptcentral.com). Register or log in to Manuscript Central, and go to the Author Center. Follow the instructions there, and select the feature topic "May 2007/Wireless Broadband Access." Questions should be directed to the guest editors.

SUBMISSION SCHEDULE

Manuscripts due:

October 15, 2006

Notification of acceptance: January 15, 2007

Final manuscript due: March 1, 2007

Publication date: May 2007

GUEST EDITORS

Dr. Mohsen Guizani

Western Michigan University

mguizani@cs.wmich.edu
Dr. Mounir Hamdi Hong Kong University of

Science and Technology hamdi@cs.ust.hk
Dr. Pascal Lorenz

University of Haute Alsace lorenz@ieee.org
Dr. Maode Ma

Nanyang Technological University

Maode_Ma@pmail.ntu.edu.sg 